\title{
Relation between Helmholtz-Kohlrausch Effect, Purity Discrimination, and Go Function
}

\author{
Yoshinobu NAYATANI \\ Osaka Electro-Communication University, \\ 18-8 Hatsu-cho, Neyagawa, Osaka 572, JAPAN

\section{Hiroaki SOBAGAKI} \\ Electrotechnical Laboratory, Life Electronics Research Center, \\ 3-11-46 Nakoji, Amagasaki, Hyogo 661, JAPAN

\section{Kenjiro HASHIMOTO} \\ Matsushita Electric Industrial Co., L.td. \\ 3-1-1 Yakumo-Nakamachi, Moriguchi, Osaka 570, JAPAN
}

Received November 17, 1993.

\begin{abstract}
Using the nonlinear color-appearance model, predicted are the four kinds of color-perception phenomena, which are the Go function studied by Evans, Munsell Values for different hues, purity discrimination, and the HelmholtzKohlrausch effect. The analysis confirmed that all the four phenomena are caused by the same common factor, the chromatic strength of spectral color stimulus reported by Evans in 1967.
\end{abstract}

\section{Introduction}

The Helmholtz-Kohlrausch effect (sometimes called brightness/luminance ratio effect for illuminant colors or lightness/luminance-factor ratio effect for object colors), ${ }^{1,2)}$ purity discrimination ${ }^{3,4)}$ and zero-gray point Go by Evans ${ }^{5)}$ are completely different color perceptions each other. However, the experimental results reported so far ${ }^{1-5)}$ suggest that mutually very close relations exist between the three phenomena. On the lightness/luminance-factor $(\mathrm{L} / \mathrm{Y})$ ratio effect, its experimental results ${ }^{1-7)}$ were well predicted ${ }^{89}$ ) within all the chromaticity gamut including spectral color stimuli by using the nonlinear color-appearance model ${ }^{10,11)}$ and the method for deriving the perceived lightness of chromatic colors ${ }^{8)}$ by the present authors.

The present paper shows that the other two phenomena are also predicted by using the nonlinear color-appearance model with a sufficiently good accuracy. In the study process, the present authors recognized the importance of Evans' claim that his Go function is a very fundamental phenomena in color-appearance study. Further, based on this Go function the reason was made clear that Munsell Values of saturated colors are high in $5 Y$ Hue and low for 5R, 5p, and 5B Hues.

In addition, the present authors also reconfirmed the other claim by Evans that the above various phenomena are illustrated commonly by the change of chromatic strength of spectral color by changing its wavelength ${ }^{5,12)}$.

\section{Go Function}

Evans reported the following experimental results on color appearance of related colors ${ }^{5}{ }^{12}$. Each of spectral color stimuli was presented to the aperture with $1^{\circ}$ visual angle at the center of white background with $10^{\circ}$ visual field. The stimuli studied consist of spectral colors and colors on the red-purple locus. The background was illuminated by light source with $7,143 \mathrm{~K}$, and its luminance was kept at $100 \mathrm{~mL}\left(\simeq 320 \mathrm{~cd} / \mathrm{m}^{2}\right)$.

The experiment was to ask each subject for assessing the color appearance of the test color stimulus presented at the center by raising its luminance from zero value. Its color appearance was perceived as an object color mode. 
When the luminance of the test stiimulus was set at zero, it was perceived as black. By increasing its luminance from zero, the blackness in its color appearance decreased, and its chroma and grayness perception appeared. Further, by increasing the luminance of the test stimulus, Evans determined its specific luminance value Go, at which just the grayness vanished and only the chroma perception existed in the color appearance of the test stimulus. Below the Go luminance, the test stimulus showed an object color mode of appearance, and the lightness perception was recognized in it.

An additional increase of the luminance of the test stimulus beyond the Go value, the test stimulus was perceived as a fluorescent color. Evans named this state fluorence. The Go luminance value of a spectral stimulus gave a critical point between the object color mode and the fluorence in its color appearance. Evans named this critical luminance value the zero-gray point corresponding to the zero grayness in the color appearance of the test stimulus, and specified the Go value by eq. (1).

$$
\mathrm{Go}=\log (\mathrm{Lo} / \mathrm{Lt})
$$

where Lo is the luminance of the background and Lt is that of the test stimulus at the point of Go.

The experimental results by Evans himself ${ }^{5}$ is shown in Fig. 1 by a dotted line from $400 \mathrm{~nm}$ to $700 \mathrm{~nm}$. The results show the minimum Go value near $570 \mathrm{~nm}$ to 580 $\mathrm{nm}$. This means the value Lt is high for yellow hue and low for red, purple, and blue hues at the Go point for a constant background luminance Lo.

Now, we will predict this Go point by using the nonlinear color-appearance model. The computed condi-

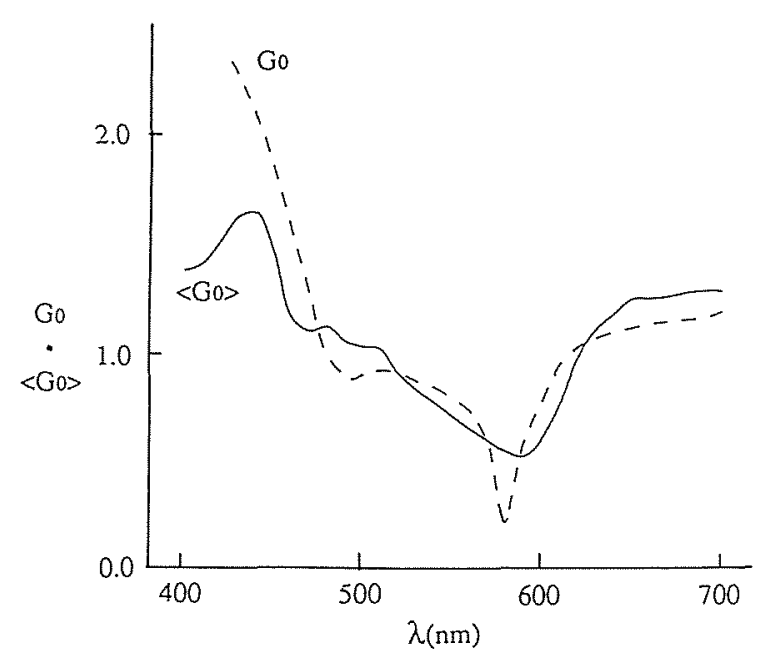

Fig. 1 Comparison between Go and < Go $>$. Dashed line corresponds to Go, and real line to $<$ Go $>$. tions are as follows; the white background with $Y_{0}=100$, illuminant $C$ for illumination, test illuminance $\mathrm{Eo}=1000$ lx (background luminance Lo $=320 \mathrm{~cd} / \mathrm{m}^{2}$ ) corresponding to the experiment by Evans, the normalizing illuminance of the nonlinear color-appearance model Eor $=1000$ $\mathrm{l} x$, and the 22 pseudo object colors with $\mathrm{Y}=0.581$ to 120.0 at each wavelength from $400 \mathrm{~nm}$ to $700 \mathrm{~nm}$ with every 10 $\mathrm{nm}$ interval. The $22 Y$ values are shown in Table 1 . The use of the above pseudo object colors corresponds to the presentation of the spectral stimuli with the luminance $L$ by eq. (2) to the central test aperture in the actual experiment.

Table 1 Twenty two $Y$ values used for deriving $<$ Go $>$

\begin{tabular}{|r|r|}
\hline NO. & $Y$ \\
\hline 1 & 0.581 \\
2 & 1.210 \\
3 & 2.021 \\
4 & 3.162 \\
5 & 4.614 \\
6 & 6.555 \\
7 & 9.003 \\
8 & 12.000 \\
9 & 15.570 \\
10 & 19.770 \\
11 & 24.580 \\
12 & 30.050 \\
13 & 36.200 \\
14 & 43.060 \\
15 & 50.680 \\
16 & 59.100 \\
17 & 68.400 \\
18 & 78.660 \\
19 & 90.010 \\
20 & 100.000 \\
21 & 110.000 \\
22 & 120.000 \\
\hline & \\
& \\
\hline $100 \pi$ & \\
&
\end{tabular}

As expected, a caution should be made to illuminate the background not to affect the luminance of the central test stimulus. This can be easily done in the actual experiment.

The computational procedure is given below.

(1) The values of Qt and Ct of test stimulus are computed by changing $X$ value from 0.581 to 120.0 at each wavelength using the nonlinear color-appearance model. The $\mathrm{Qt}$ function is a correlate to the achromatic component of lightness of the test color, and the $\mathrm{Ct}$ function corresponds to its 


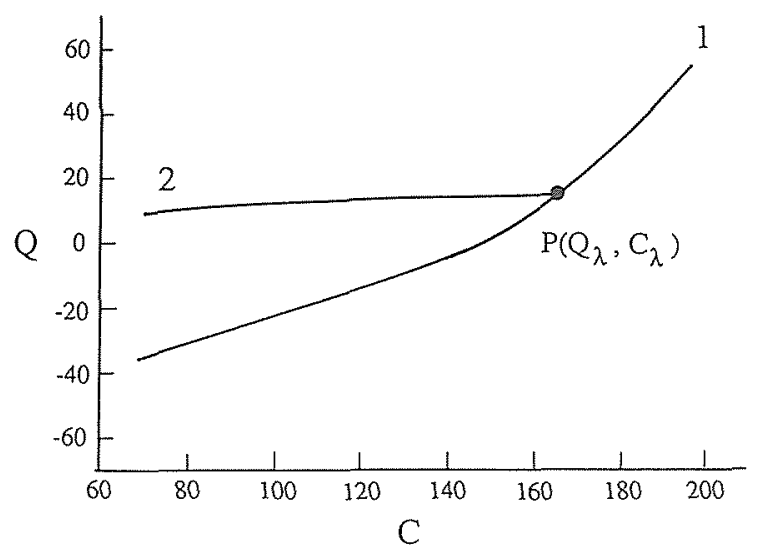

Fig. 2 Determination of the point $P$ with $Q_{\lambda}, C_{\lambda}$, corresponding to $<\mathrm{Go}>$ at $550 \mathrm{~nm}$. The curve 1 corresponds to the relation between $Q_{\tau}$ and $C_{t}$, and the curve 2 to the relation between $Q_{v}\left(C_{t}\right)$ and $C_{t}$. The point $P$ is the intersection between the two carves 1 and 2 .

chroma. The computed results are shown in a Q$\mathrm{C}$ plane. An example is shown in Fig. 2 to $550 \mathrm{~nm}$. The curve 1 in the figure corresponds to the $Q t-$ $\mathrm{Ct}$ curve at $550 \mathrm{~nm}$. For the specified illumination and background, the curve 1 corresponds to the maximum chroma perception at any specified value of $\mathrm{Qt}$ for each test stimulus. This means that the spectral stimulus at $550 \mathrm{~nm}$ can not have chroma perceptions in the right hand side of curve 1.

(2) At each wavelength, the locus is specified by eq. (3), on which only grayness and chroma perceptions coexist"

$\mathrm{Qv}(\mathrm{Ct})=\mathrm{q}_{1}(\theta) \mathrm{Ct}+\mathrm{q}_{2}(\theta) \mathrm{C}_{\mathrm{t}}^{2} \times 10^{-4}$,

where $\theta$ is the hue angle in the nonlinear colorappearance model, and the functions $\mathrm{q}_{1}(\theta)$ and $\mathrm{q}_{2}(\theta)$ are shown in Appendix $C$ of our previous article The values of $\theta$ are also given in Table 2 for each wavelength $\lambda$. The object colors predicted by eq. (3) correspond to those found on the horizontal line ${ }^{13)}$ connecting gray $(s=50, C r=100)$ and the full color $(s=0, C r=100)$ at each of the NCS constant hue planes. ${ }^{14)}$ Though the NCS gray has Munsell Value 5.8/, we specified the gray point by Munsell Value 5/ in the non-linear color-appearance model, and the grayness-chroma curve in eq. (3) is specified by the gray with $5 /$ as a starting point. This selection was done by con-
Table 2 Relation between $\lambda$ and $\theta^{\circ}$

\begin{tabular}{|c|c|c|c|}
\hline$\lambda(\mathrm{nm})$ & $\theta^{\circ}$ & $\lambda(\mathrm{nm})$ & \multicolumn{1}{c|}{$\theta^{\circ}$} \\
\hline 400 & 310.3 & 600 & 68.09 \\
410 & 306.9 & 610 & 56.61 \\
420 & 299.2 & 620 & 48.87 \\
430 & 279.3 & 630 & 44.13 \\
440 & 253.7 & 640 & 40.92 \\
450 & 232.9 & 650 & 39.02 \\
460 & 220.2 & 660 & 37.89 \\
470 & 213.9 & 670 & 37.33 \\
480 & 207.2 & 680 & 36.94 \\
490 & 194.1 & 690 & 36.66 \\
500 & 176.1 & 700 & 36.57 \\
510 & 159.1 & -565 & 330.10 \\
520 & 145.9 & -560 & 337.35 \\
530 & 137.9 & -550 & 342.30 \\
540 & 131.6 & -540 & 344.71 \\
550 & 125.6 & -520 & 347.88 \\
560 & 118.7 & -505 & 351.87 \\
570 & 109.6 & -500 & 355.12 \\
580 & 97.5 & -497 & 358.99 \\
590 & 82.7 & -495 & 4.03 \\
& & -494 & 8.68 \\
& & -493 & 18.18 \\
\hline
\end{tabular}

sidering the achromatic color with 5/ the gray.

The curve 2 in Fig. 2 shows the relation between $\mathrm{Qv}(\mathrm{Ct})$ and $\mathrm{Ct}$. Moving along the $\mathrm{Qv}(\mathrm{Ct})-\mathrm{Ct}$ curve to the right direction, the grayness decreases and to the contrary the chroma increases in the color appearance of the object colors on it.

(3) The values of $Q_{\lambda}, C_{\lambda}$ of the point $P$ in Fig. 2 is determined corresponding to the intersection of curves 1 and 2 in Fig. 2. The point P corresponds to the color perception with the maximum chroma but with the minimum grayness in the test color at each wavelength. The values $Q_{\lambda}$ and $C_{\lambda}$ are considered the Go point at the wavelength $\lambda$ under consideration. They were estimated from $400 \mathrm{~nm}$ to $700 \mathrm{~nm}$, and are shown in Table 3.

(4) The values of $Q_{\lambda}$ and $C_{\lambda}$ in Table 3 are further transformed to colorimetric values $\left\langle\mathrm{x}_{\lambda}\right\rangle_{,}\left\langle\mathrm{y}_{\lambda}\right\rangle, \mathrm{Y}_{\lambda}$ by using inverse transformation of the nonlinear color-appearance model. In the transformation, again illuminant $C, Y_{0}=100$, and Eo $=$ Eor $=1000$ $\mathrm{lx}$ are used. By confirming the sufficiently well agreement between the derived $\left\langle\mathrm{x}_{\lambda}\right\rangle,\left\langle\mathrm{y}_{\lambda}\right\rangle$ values and the input $x_{\lambda}, y_{\lambda}$ values in step 1, the check of the whole computations done were made. The fourth column of Table 3, the values of $Y_{\lambda}$ are shown. 
Table 3 Values of $Q_{\lambda}, C_{\lambda}, Y_{\lambda}$, and $\log \left(100 / Y_{\lambda}\right)$ corresponding to the $<$ Go $>$ values for spectral colors

\begin{tabular}{|c|c|c|c|c|}
\hline$\lambda$ & $Q_{\lambda}$ & $\mathrm{C}_{\lambda}$ & $Y_{\lambda}$ & $\log \left(100 / Y_{\lambda}\right)$ \\
\hline 400 & -14.0 & 292.0 & 7.67 & 1.12 \\
\hline 410 & -15.5 & 282.0 & 7.14 & 1.15 \\
\hline 420 & -20.0 & 262.0 & 5.66 & 1.25 \\
\hline 430 & -26.0 & 229.0 & 4.39 & 1.36 \\
\hline 440 & -29.0 & 219.0 & 4.24 & 1.37 \\
\hline 450 & -25.0 & 248.0 & 6.43 & 1.19 \\
\hline 460 & -16.0 & 280.0 & 12.76 & 0.89 \\
\hline 470 & -13.0 & 255.0 & 14.81 & 0.83 \\
\hline 480 & -12.0 & 199.0 & 14.14 & 0.85 \\
\hline 490 & -8.0 & 158.0 & 16.49 & 0.78 \\
\hline 500 & -6.0 & 140.0 & 17.49 & 0.76 \\
\hline 510 & -5.0 & 142.5 & 17.92 & 0.75 \\
\hline 520 & 1.0 & 153.5 & 23.19 & 0.63 \\
\hline 530 & 6.0 & 150.5 & 27.63 & 0.56 \\
\hline 540 & 11.0 & 164.0 & 31.87 & 0.50 \\
\hline 550 & 16.5 & 160.5 & 36.81 & 0.43 \\
\hline 560 & 22.0 & 163.5 & 42.37 & 0.37 \\
\hline 570 & 27.5 & 156.5 & 48.13 & 0.32 \\
\hline 580 & 33.0 & 148.0 & 54.24 & 0.27 \\
\hline 590 & 36.0 & 139.0 & 56.90 & 0.24 \\
\hline 600 & 31.0 & 140.5 & 47.00 & 0.33 \\
\hline 610 & 20.0 & 144.5 & 32.43 & 0.49 \\
\hline 620 & 7.0 & 148.5 & 19.80 & 0.70 \\
\hline 630 & -0.5 & 156.0 & 14.58 & 0.84 \\
\hline 640 & -4.5 & 163.5 & 12.31 & 0.91 \\
\hline 650 & -8.5 & 168.0 & 9.94 & 1.00 \\
\hline 660 & -8.5 & 172.0 & 9.92 & 1.00 \\
\hline 670 & -9.0 & 173.5 & 9.62 & 1.02 \\
\hline 680 & -10.0 & 175.0 & 9.04 & 1.04 \\
\hline 690 & -10.5 & 175.5 & 8.77 & 1.06 \\
\hline 700 & -10.5 & 176.0 & 8.76 & 1.06 \\
\hline
\end{tabular}

(5) At each wavelength, the $<$ Go $>$ values, the estimates of Go, are given by eq. (4).

$<$ Go $>=\log \left[100 / Y_{\lambda}\right]$

The value 100 corresponds to the tristimulus value $Y$ of the background. The value $100 / Y_{\lambda}$ in eq. (4) corresponds to Lo/Lt in eq. (1). The values of $<$ Go $>$ are shown in the fifth column of Table 3 . The real line in Fig. 1 corresponds to the $<$ Go $>$ values. Its height was adjusted by using the method of least squares for $400 \mathrm{~nm}$ to $700 \mathrm{~nm}$ to have a good conformity between Go and < Go $\rangle$.

By inspecting Fig. 1, the agreement between Go and $<$ Go $>$ is pretty good except for the wavelength region shorter than $440 \mathrm{~nm}$ and the steep change of Go near $580 \mathrm{~nm}$. The observed Go curve in Fig. 1 is based upon experiment only done by Evans himself. In addition, the accurate visual observation is generally difficult in the spectral range less than $440 \mathrm{~nm}$. Considering a pretty good agreement $t^{9)}$ between the experimental results and the predictions in the Helmholtz-Kohlrausch effect for the spectral range, the cause of the deviation between Go and < Go > below $440 \mathrm{~nm}$ can not be reduced to only the predicting method of $<$ Go $>$ used. In fact, the originally measured value of $G 0$ by Evans ${ }^{12)}$ gave a saturated characteristic instead of a uniform increase to high values as shown in the dotted line in Fig. 1 below $440 \mathrm{~nm}$.

Next we consider the reason of the deviation between Go and < Go > near $580 \mathrm{~nm}$. The Qv (Ct) function in eq. (3) used for estimating the $<\mathrm{Go}>$ function is derived from the analysis of object colors between gray and full color at each hue plane of the NCS color scheme. However, the steep change of $\mathrm{Qv}(\mathrm{Ct})$ function was not found near $580 \mathrm{~nm}$ in the NCS color scheme. This is the reason why the $<$ Go $>$ function did not show the steep change near $580 \mathrm{~nm}$.

Now, we consider the meaning of Go or Lo/Lt. The quantity $L t$ is the minimum luminance needed to cancel the blackness found in the test color stimulus at $\mathrm{Lt}=0$ completely. The color perception of the test stimulus at Go consists of only chroma component. The Lt value is high near 580 $\mathrm{nm}$ (yellow hue) and low near $450 \mathrm{~nm}$ and 650 $\mathrm{nm}$. The Go value changes to the contrary to Lt. The characteristic of Go function suggests that yellow spectral color stimuli are weaker in the power for canceling the blackness perceptions existing in them than those existing in other hues.

This canceling power corresponds to the chromatic strength of test color stimulus reported by Evans $^{12)}$.

\section{Y Values of Full Colors in Munsell Color Scheme}

The Munsell Values of full colors are different for different Munsell Hues. Approximately, they are estimated $5 /$ to $5 R, 9 /$ to $5 Y, 5 /$ to $6 /$ to $5 G$, and $3 /$ to $4 /$ to $5 \mathrm{~PB}^{15)}$. Evans ${ }^{12,16)}$ already stated that the differences of Munsell Values of full colors are caused by the change of chromatic strength for different Hues as found from the Go function in Fig. 1. As the chromaticity coordinates of full colors in the above 4 Munsell-Hue planes are very close to those of spectral colors, the following spectral colors were substituted for the above Munsell full colors; $700 \mathrm{~nm}$ for $5 \mathrm{R}, 580 \mathrm{~nm}$ for $5 Y, 510 \mathrm{~nm}$ for $5 \mathrm{G}$, and $400 \mathrm{~nm}$ for $5 \mathrm{~PB}$. The values $Y_{\lambda}$ for the 4 wavelengths in Table 3 were specified as the $Y$ values of full colors in the four Munsell Hues considered. The value $Y_{\lambda}$ corresponds to the $<$ Go $>$ value directly. The results of $Y(V /)$ estimated are $8.8(3.5 /)$ for $5 R, 54.2(7.7 /)$ for $5 Y, 17.9(4.8 /)$ for $5 G, 7.6$ 
(3.2/) for 5PB. The numbers in the parenthness show the Munsell Values V/ of the estimated $Y$ value.

The Munsell Values of full colors for the four Munsell Hues predicted from $<\mathrm{Go}>$ change similarly to those already shown in the Munsell scheme. A detailed inspection shows that the predicted Munsell Values are uniformly about 1 Value lower than those found in the Munsell scheme. The cause of the above deviations between the predicted and the specified Munsell Values may be caused by the use of Munsell Value 5/ for the complete gray in the nonlinear color-appearance model. The gray of the NCS scheme ( $s=50, \mathrm{Cr}=0$ ) estimated by using a white background has the Munsell Value 5.8/. If the NCS gray point is used for the gray point in the nonlinear color appearance model, the Value estimates for full colors from < Go > increase by about 0.8 Value. This may eliminates the above 1 Munsell Value deviations.

\section{Purity Discrimination}

We consider that a spectral color stimulus is added to an achromatic one. The total luminance of the mixed color stimulus is always kept constant by adjusting the luminance of achromatic stimulus irrespective of the added quantity of spectral color stimulus. When the luminance of added spectral color stimulus is very little, the mixed color stimulus is still perceived as achromatic. By adding an appreciable amount of the spectral color stimulus, the chromatic component in the mixed color stimulus is clearly perceived corresponding to the hue of the added spectral color. In an intermediate amount of mixing spectral color stimulus, the purity threshold exists.

The purity threshold $\Delta \mathrm{PC}_{\lambda}$ is given by eq. (5).

$$
\Delta P c_{\lambda}=\Delta L_{\lambda} /\left(L_{w}+\Delta L_{\lambda}\right)
$$

where $\Delta L_{\lambda}$ is the luminance of the added spectral color stimulus at the purity threshold, and Lw is the luminance of achromatic color stimulus. As already stated the total luminance $\left(\mathrm{LW}+\Delta \mathrm{L}_{\lambda}\right)$ is kept constant throughout in the experiment.

Many studies were reported so far in the purity threshold $^{3,4)}$. Pridmore ${ }^{4)}$ summarized the results of various experimental and theoretical studies on the purity discrimination. Figure 3 shows the experimental results corresponding to the two kinds of different experimental conditions.

The curve 1 in Fig. 3 corresponds to the puritythreshold experiment reported by Priest et al. ${ }^{17}$. The curve 2 in the figure corresponds to the purity difference on the equi-chroma colors, which are experimentally determined by Uchikawa et al. ${ }^{18)}$ in the color gamut beyond the purity threshold. In other words, the curve 1 in Fig. 3 may corresponds to the equi-chroma loci at the purity

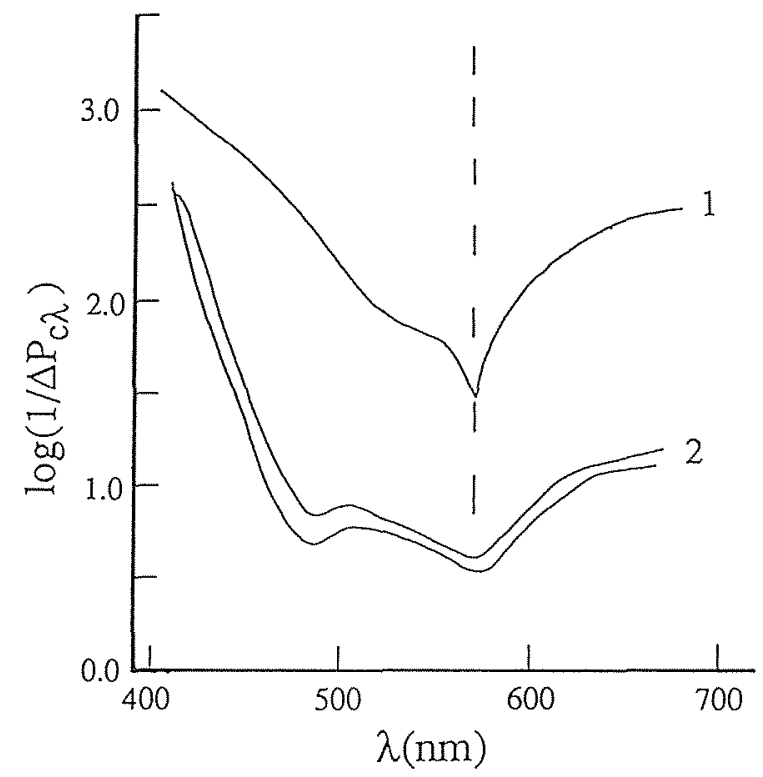

Fig. 3 Two kinds of discrimination curves on colorimetric purity. The curve 1 corresponds to the discrimination experiment at threshold, and the curve 2 to the experiment estimating a constant perceived chroma above the threshold.

threshold. It is also believed that the difference exists between the color-differences at and beyond the colordifference threshold. On the purity discrimination, the differences at and beyond the purity threshold are found as shown in curves 1 and 2 in Fig. 3.

The two curves in Fig. 3 generally agree in shape. However, its detailed inspection clarifies that the purity discrimination at threshold (curve 1) changes steeper than that beyond threshold (curve 2) in the spectral range 500 $\mathrm{nm}$ to $570 \mathrm{~nm}$.

The nonlinear color-appearance model does not expect to predict the color discrimination at threshold. By this reason, the colorimetric purity curve was estimated to the object colors corresponding to an equi-chroma perception beyond the threshold using the model. The predicted results are expected to be similar to the experimental results by Uchikawa et al. ${ }^{18)}$ (curve 2 of Fig. 3).

The conditions of computation are as follows; $Y$ value of achromatic background $Y_{O}=20$, illuminant $C$ for illumination, Eo $=$ Eor $=1000 \mathrm{~lx}$. The test samples are the object colors with $Q=0$ and $C=4$ in the nonlinear colorappearance space with different dominant wavelengths.

The following proceciure was used for computation.

(1) To each dominant wavelength $\lambda$ (its hue angle $\theta_{\lambda}$ ), the following quantities are specified. 
$Q=0$,

$\mathrm{T}=\mathrm{C} \cos \theta_{\lambda}$

$\mathrm{P}=\mathrm{C} \sin \theta_{k}$

where $C=4$. The relation between $\lambda$ and $\theta_{\lambda}$ is already given in Table 2.

(2) To each wavelength, the values $Q, T, P$ in eq. (6) are further transformed to the corresponding

Table 4 Values of $x_{\lambda}^{\prime}, y_{\lambda}^{\prime}$, and $\log \left[1 / \Delta P_{c \lambda}\right]$ corresponding to the equal chroma locus with $Q=0$, $C=4$ (Munsell colors with $5 / 0.7$ ) in the nonlinear color-appearance model.

\begin{tabular}{|c|c|c|c|}
\hline$\lambda$ & $x_{\lambda}^{\prime}$ & $y_{\lambda}^{\prime}$ & $\log \left[1 / \Delta P_{\mathrm{c} \lambda}\right]$ \\
\hline 400 & 0.3078 & 0.3052 & 3.26 \\
410 & 0.3072 & 0.3050 & 3.25 \\
420 & 0.3060 & 0.3048 & 3.21 \\
430 & 0.3034 & 0.3051 & 3.09 \\
440 & 0.3015 & 0.3074 & 2.99 \\
450 & 0.3013 & 0.3102 & 2.94 \\
460 & 0.3013 & 0.3120 & 2.86 \\
470 & 0.3014 & 0.3130 & 2.64 \\
480 & 0.3017 & 0.3141 & 1.79 \\
490 & 0.3025 & 0.3166 & 1.57 \\
500 & 0.3044 & 0.3203 & 1.50 \\
510 & 0.3069 & 0.3237 & 1.40 \\
520 & 0.3093 & 0.3262 & 1.30 \\
530 & 0.3109 & 0.3277 & 1.24 \\
540 & 0.3123 & 0.3287 & 1.18 \\
550 & 0.3137 & 0.3296 & 1.13 \\
560 & 0.3153 & 0.3305 & 1.07 \\
570 & 0.3175 & 0.3314 & 0.97 \\
580 & 0.3204 & 0.3321 & 1.13 \\
590 & 0.3231 & 0.3312 & 1.20 \\
600 & 0.3242 & 0.3286 & 1.30 \\
610 & 0.3244 & 0.3261 & 1.39 \\
620 & 0.3242 & 0.3243 & 1.45 \\
630 & 0.3239 & 0.3231 & 1.50 \\
640 & 0.3237 & 0.3223 & 1.54 \\
650 & 0.3235 & 0.3219 & 1.56 \\
660 & 0.3234 & 0.3216 & 1.57 \\
670 & 0.3234 & 0.3214 & 1.58 \\
680 & 0.3233 & 0.3213 & 1.59 \\
690 & 0.3233 & 0.3213 & 1.59 \\
700 & 0.3233 & 0.3212 & 1.59 \\
-565 & 0.3115 & 0.3071 & 2.74 \\
-560 & 0.3130 & 0.3081 & 2.39 \\
-550 & 0.3140 & 0.3089 & 2.17 \\
-540 & 0.3145 & 0.3093 & 2.07 \\
-520 & 0.3151 & 0.3099 & 1.95 \\
-505 & 0.3159 & 0.3106 & 1.84 \\
-500 & 0.3166 & 0.3113 & 1.78 \\
-497 & 0.3174 & 0.3121 & 1.77 \\
-493 & 0.3184 & 0.3132 & 1.74 \\
& 0.3193 & 0.3142 & 1.71 \\
\hline & 0.3210 & 0.3166 & 1.66 \\
\hline
\end{tabular}

chromaticity coordinates $x_{\lambda}^{\prime}, y_{\lambda}^{\prime}$ by using the inverse transformation of the nonlinear color-appearance model. The results are shown in Table 4.

The locus of the derived chromaticity coordinates is shown by a real line in Fig. 4, and that of the estimated chromaticity coordinates of color samples with $5 / 0.7$ is shown by a dashed line. As expected, the both curves are similar to each other.

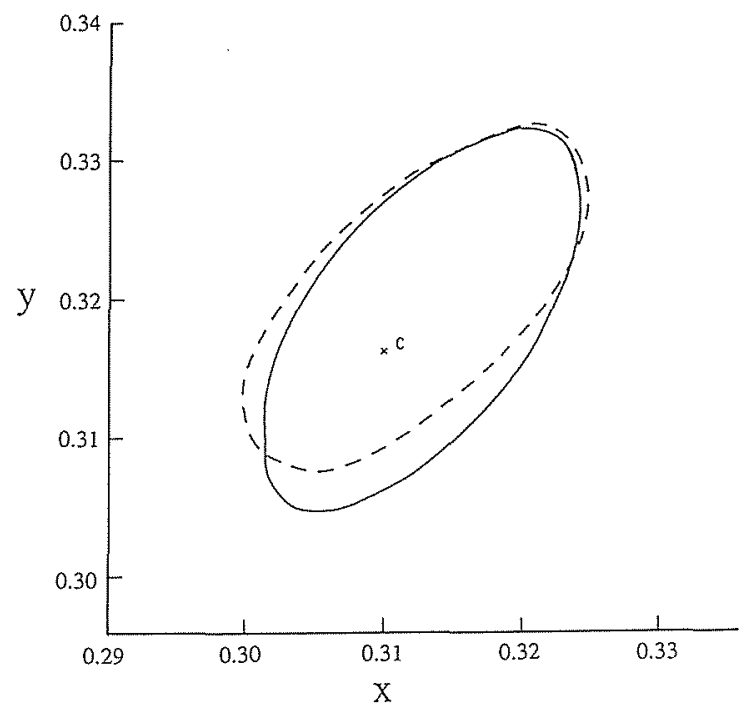

Fig. 4 Comparison between the chromaticity locus of the object colors with $Q=0, C=4$ (real line) and that of the Munsell colors with $5 / 0.7$ (dashed line)

(3) The value of $\triangle P \mathrm{PC}_{\lambda}$ is derived by eq. (7). It is already well known that eq. (5) is completely equivalent to eq. (7).

$\Delta P C_{\lambda}=y^{d} \lambda_{\lambda} \times P e / y^{\prime}$,

where $y^{d} \lambda$ is the $y$ chromaticity coordinate of dominant wavelength $\lambda$, and $P e$ is the excitation purity of $x_{\lambda}^{\prime}, y^{\prime}$.

(4) Derivation of $\log \left(1 / \Delta P_{C_{2}}\right)$. The computed results are also shown in Table 4 . Figure 5 shows the values of $\log \left(1 / \Delta P_{c_{\lambda}}\right)$ from $400 \mathrm{~nm}$ to $700 \mathrm{~nm}$, and the results are quite similar to curve 2 in Fig. 3 as expected.

It is not a good agreement between Fig. 5 and curve 1 of Fig. 3 , because the nonlinear color-appearance model does not expect to predict color discrimination at threshold. This insufficient agreement may suggest the necessity of adding some mechanisms to the nonlinear color-appearance model to make it possible to predict the discrimination phenomena at threshold. 


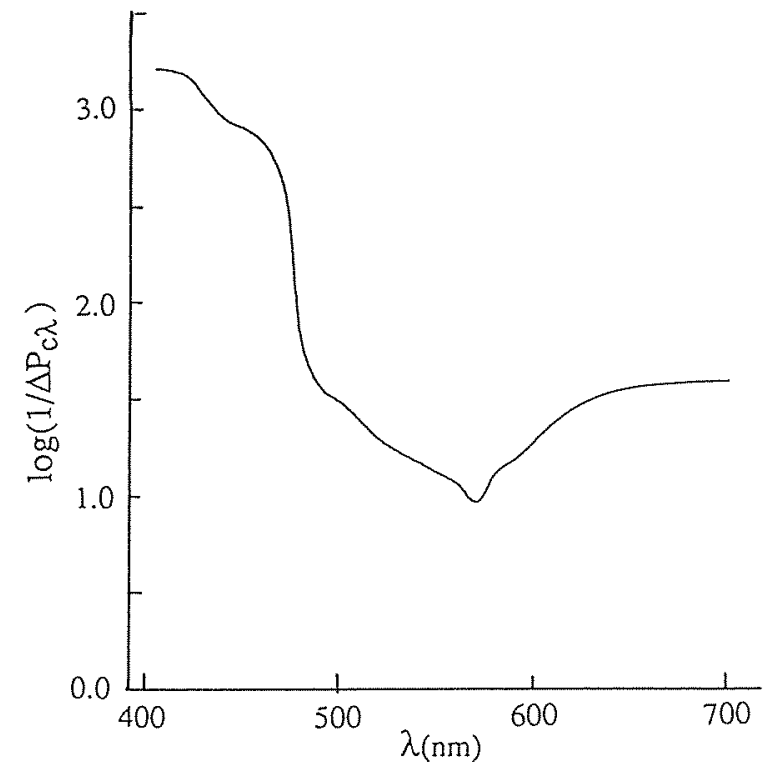

Fig. 5 Relation belween colorimetric purities for object colors with $\mathrm{Q}=0$ and $\mathrm{C}=4$ and their dominant wavelengths.

The Go function in Fig. 1 and the purity discrimination in Fig. 3 are completely different phenoma, and they were estimated by using completely different experimental methods. Even so, spectral characteristics of the two phenomena are quite similar. Evans ${ }^{12)}$ already pointed out that the functions $G o$ and $\log \left(1 / \Delta P_{C \lambda}\right)$ could be illustrated by the wavelength characteristic of the chromatic strength of spectral color stimuli and were closely related each other.

As clearly found in Figs. 3 and 5 , the low value of $\log$ $\left(1 / \Delta \mathrm{P}_{\mathrm{C} \lambda}\right)$ in the range $570 \mathrm{~nm}$ to $580 \mathrm{~nm}$ naturally suggests that $\Delta L_{\lambda}$ is high as compared with other wavelengths for constant value of $L w+\Delta L_{\lambda}$. To the contrary, $\Delta L_{\lambda}$ is low for the spectral range near $400 \mathrm{~nm}$. In other words, the chromatic strength of yellow hue spectral stimuli are weaker than those with other hues, for example blue, purple and red hues. In the present paper, this Evans' insight was theoretically confirmed by predicting the two phenomena by using the single nonlinear color-appearance model.

\section{Helmholtz-Kohlrausch Effect}

The luminance of each spectral color stimulus is determined by the flicker photometry and the CIE 1924 spectral luminosity function $V(\lambda)^{20)}$ is effective to compute the luminance value of any color stimulus. However, two spectral color stimuli kept at the same lumi- nance but with different wavelengths are generally different in brightness. For example, the brightness of 450 $\mathrm{nm}$ color stimulus is higher than that of $570 \mathrm{~nm}$ one in their direct comparison, even though they are kept at the same luminance. By this reason, CIE reported the luminous efficiency function $V_{b, 2}(\lambda)$ for heterochromatic brightness matching in $1988^{2)}$.

The deviations between $V_{b, 2}(\lambda)$ and $V(\lambda)$ correspond to the Helmholtz-Kohlrausch effect or brightness/luminance $(B / L)$ ratio effect for luminous colors. Several experimental studies are given in the reference of the technical report ${ }^{2)}$.

Based on these studies, the $B / L$ ratio effect is estimated by eq. (8) below. However, in eq. (8), the Judd modified spectral luminosity function $V_{M}(\lambda)$ is used instead of $V(\lambda)$. The $V_{M}(\lambda)$ function initially reported by Judd ${ }^{21)}$ and recommended its final version by CIE in 1990, because of the shortage in sensitivity of the $V(\lambda)$ function for the spectral range less than $460 \mathrm{~nm}$.

$$
\begin{aligned}
\delta(\lambda)= & \log \left[V_{b, 2}(\lambda) / V_{M}(\lambda)\right]- \\
& \log \left[V_{b, 2}(570) / V_{M}(570)\right]
\end{aligned}
$$

The value of $\delta(\lambda)$ is normalized to zero at $570 \mathrm{~nm}$. The computed results by eq. (8) is shown by the real line in Fig. 6. The dotted and the dott-dash line in Fig. 1 are the predicted lightness / luminance factor $(\mathrm{L} / \mathrm{Y})$ ratios $^{9)}$ by using the nonlinear color-appearance model ${ }^{10,11)}$ and the hypothesis $^{8)}$ on the perceived lightness of chromatic object colors. They give the estimates of the $B / L$ ratios of the real line on the same spectral range. The dotted line in Fig. 6 is derived on the basis of the Wyszecki experi-

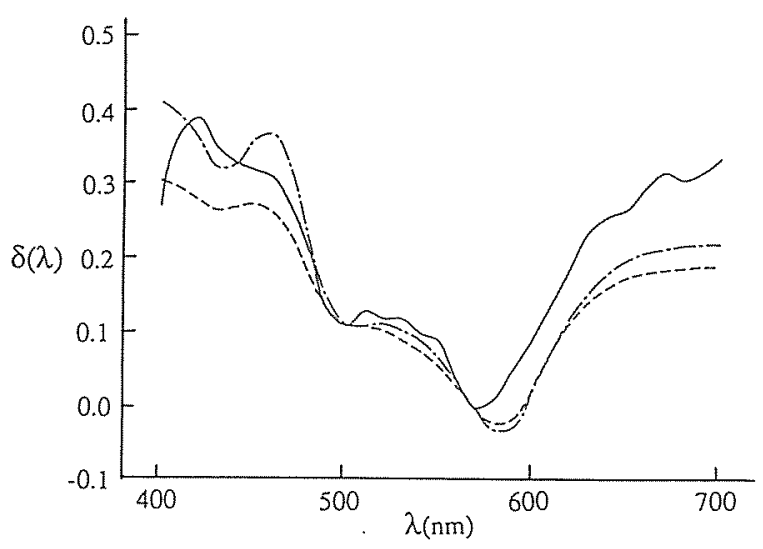

Fig. 6 Three kinds of estimations on the HelmholtzKohlrausch effect. Real line corresponds to $\delta(\lambda)$ in eq. (7). Dot-dash line is estimated on the basis of the Wyszecki experiments ${ }^{6}$, and dashed line on the basis of the Sanders-Wyszeeki experiment? 
ment ${ }^{6)}$ using real object colors, and the dott-dash line is derived on the basis of the Sanders-Wyszecki experiment ${ }^{7)}$ using pseudo-object colors: The agreement between the three curves in Fig. 6 is remarkably good. Again, the Helmholtz-Kohlrausch effect is high near $400 \mathrm{~nm}$ and $700 \mathrm{~nm}$ as compared with near $570 \mathrm{~nm}$. This again shows that the chromatic strength is low at near $570 \mathrm{~nm}$ as compared with other wavelengths.

The chromatic strength is the ability of spectral color stimulus to cancel the blackness in the Go function, and to the contrary in the Helmholtz-Kohlrausch effect the chromatic strength of spectral color stimulus is the ability to induce the brightness or the lightness perception. This two concepts on the chromatic strength are considered to be equivalent.

It should be noted that the effect in Fig. 6 is relatively smaller (about one third) than those found in Fig. 1 on the same logarithmic scale. This is easily found by comparing the effect difference between $400 \mathrm{~nm}$ and 570 $\mathrm{nm}$ in the figures. At present, the authors suspect that the small magnitude of the Helmholtz-Kohlrausch effect found in Fig. 6 is mainly caused by the method used for representing the effect. On this problem, we will discuss in our future study.

\section{Conclusion}

The conclusions in the present studies are summarized as follows.

(1) It was made clear that all the different four phenomena, the Go function, the change of Munsell Values of full colors for different hues, purity discrimination, and the Helmholtz-Kohlrausch effect, are caused by the same common factor, the chromatic strength of spectral color stimulus already reported by Evans ${ }^{3,12)}$ in 1967.

(2) The chromatic strength of spectral colors near 570 $\mathrm{nm}$ is lower than other spectral colors. This means that the ability for canceling the achromatic component (blackness) found in color stimulus is low in the spectral colors near $570 \mathrm{~nm}$. This situation corresponds to the Go function and the $Y$ values of most saturated colors in the Munsell scheme. For the constant chroma contour, the chromatic strength of spectral colors is understood as follows. The luminance value $\Delta \mathrm{L}_{\lambda}$ in eq. (5) at about 570 $\mathrm{nm}$ for inducing a constant chroma perception is higher (lower in chromatic strength) than other wavelengths. In the Helmholtz-Kohlrausch effect, the chromatic strength of spectral colors are found by the low ability for increasing the brightness or lightness in the color stimulus at about $570 \mathrm{~nm}$ as compared with other wavelengths.

(3) The above four phenomena in item (1) were well predicted by the nonlinear color-appearance model $^{10,11)}$ and the method for deriving the perceived lightness of chromatic colors ${ }^{8)}$. The nonlinear color-appearance model has a wide range of application including the prediction of chromatic adaptation.

(4) The authors now believe that the Go function is a fundamental quantity on the color appearance of object and luminous colors. The importance of the studies by Evans should be recognized in the colors community.

\section{Acknowledgment}

Thanks are due to Messrs. H. Takagi and T. Yamada, the former students of the Osaka Electro-Communication. University, for making laborious numerical computations needed in the present study. Also the authors wish to thank the research grant from the Illuminating Engineering Institute of Japan to the present study in 1993.

\section{References}

(1) Wyszecki G. and Stiles W.S.: Color Science, 2nd ed., John Wiley \& Sons, New York, (1984) 410-420

(2) Publication No. CIE 75, Spectral luminous efficiency functions based upon brightness matching for monochromatic sources, Central Bureau of the CIE, Vienna, (1988)

(3) Reference 1 pp. 571-574

(4) Pridmore, R.W.: Model of saturation and brightness: relations with luminance, Color Res. Appl. Vol. 15 (1990) 344-357

(5) Evans R.H.: The perception of color, John Wiley \& Sons, New York, (1974) 410-420

(6) Wyszecki G.: Correlate for brightness in terms of CIE chromaticity coordinates and luminous reflectance, J. Opt. Soc. Am. Vol. 57 (1967) 254-257

(7) Sanders C.L., and Wyszecki G.: Correlate for brightness in terms of CIE color-matching data, CIE Proceedings 15th Session, Vienna, 1963, Paper P63.6, CIE Central Bureau, Paris (1964)

(8) Nayatani Y., Umemura Y., Sobagaki H., Takahama K., and Hashimoto K.: Lightness perception of chromatic object colors, Color Res. Appl. Vol. 16 (1991) 16-25

(9) Nayatani Y., Gomi Y., Kamei M., Sobagaki H., and Hashimoto K.: Perceived lightness of chromatic object colors including highly saturated colors, Color Res. Appl. Vol. 7 (1992) 127-141

(10) Nayatani Y., Hashimoto K., Takahama K., and Sobagaki H.: A nonlinear color-appearance model using Estévez-Hunt-Pointer primaries, Color Res. Appl. Vol. 12 (1987) 231-242

(11) Nayatani Y., Takahama K., Sobagaki H., and Hashimoto K.: Color-appearance model and chromatic-adaptation transform, Color Res. Appl. Vol. 15 (1990) 210-221

(12) Evans R.M., and Swenholt B.K.: Chromatic strength of colors: dominant wavelength and purity, J. Opt. 
Soc. Am. Vol. 57 (1967) 1319-1324

(13) Nayatani Y., Umemura Y., Hashimoto K., Takahama K., and Sobagaki H.: Analyzing the Natural Color System's Color-order system by using a nonlinear color-appearance model, Color Res. Appl. Vol. 14 (1989) 69-78

(14) Håld A., and Sivik L.: NCS-Natural Color System: a Swedish standard for color notation, Color Res. Appl. Vol. 6 (1981) 129-138

(15) Newhall S.M.: D. Nickerson, and D.B. Judd, Final report of the O.S.A. subcommittee on spacing of the Munsell colors, J. Opt. Soc. Am. Vol. 33 (1943) 385418

(16) Evans R.M., and Swenholt B.K.: Chromatic strength of colors, Part II. The Munsell System, J. Opt. Soc. Am. Vol. 58 (1968) 580-584

(17) Priest I.G., and Brickwedde F.G.: The minimum perceptible colorimetric purity as a function of dominant wavelength, J. Opt. Soc. Am. Vol. 28 (1938) 133-139

(18) Uchikawa K., Uchikawa H., and Kaiser P.K.: Equating colors for saturation and brightness: the relationship to luminance, J. Opt. Soc. Am. Vol. 72 (1982) 1219-1224

(19) Publication No. CIE 86, CIE $19882^{\circ}$ spectral luminous efficiency function for photopic vision, CIE central Bureau, Vienna, (1990)

(20) CIE Proceedings 1924, Cambridge University Press, Cambridge, (1926)

(21) Judd D.B.: Report of U.S. secretariat committee on colorimetry and artificial daylight, CIE proceedings stockholm, CIE Central Bureau, paris, 1951, Vol. 1 Part 7, p. 11 